\title{
Very Compact Stacked $L C$ Resonator-Based Bandpass Filters With a Novel Approach to Tune the Transmission Zeros
}

\author{
Chien-Hsun Chen, Student Member, IEEE, Chien-Hsiang Huang, Student Member, IEEE, \\ Tzyy-Sheng Horng, Senior Member, IEEE, Sung-Mao Wu, Member, IEEE, Jian-Yu Li, Student Member, IEEE, \\ Cheng-Chung Chen, Member, IEEE, Chi-Tsung Chiu, and Chih-Pin Hung
}

\begin{abstract}
This letter presents very compact stacked $L C$ resonator structures and their coupling and excitation techniques for designing the bandpass filters operating in the $3.5 \mathrm{GHz}$ WiMAX band. Using a coupled stacked $L C$-resonator structure not only greatly reduces the overall size but also can create multiple transmission zeros to enhance roll-off rate or desired stopband rejection for the designed bandpass filters. Furthermore, this letter develops a simple technique of etching a rectangular $C$-shaped slot in the bottom ground plane to flexibly adjust the transmission-zero frequencies without changing the size and shape of the filter.
\end{abstract}

Index Terms-Bandpass filter (BPF), stacked LC resonator, transmission zeros.

\section{INTRODUCTION}

$\mathbf{M}$ INIATURIZED structure, high selectivity, and large stopband rejection are critical for wireless communication bandpass filters. Most conventional microstrip filters to date are based primarily on transmission-line structures [1]. These filters require too much area because each resonator needs quarter- or half-wavelength to get resonance. Some other studies propose novel structures to reduce filter size, including stepped impedance resonator (SIR) [2], defected ground structure (DGS) resonator [3], patch-via-spiral resonator [4], and net-type resonator [5]. However, the resulting designs are still not size-competitive with low-temperature cofired ceramic (LTCC) bandpass filters. In addition to a miniature size, the transmission zeros are widely used to improve the selectivity or the rejection at the desired stopband frequencies in bandpass filter design. The cascaded quadruplet bandpass filters [2], [5]

Manuscript received December 01, 2008; revised February 16, 2009. First published April 24, 2009; current version published May 08, 2009. This work was supported in part by the National Science Council of Taiwan, under Grants 97-2221-E-110-035-MY3 and 97-2622-E-110-007-CC1, and by the Department of Industrial Technology, Taiwan, under Grant 97-EC-17-A-01-S1-104.

C.-H. Chen, C.-H. Huang, and T.-S. Horng are with the Department of Electrical Engineering, National Sun Yat-Sen University, Kaohsiung 804, Taiwan (e-mail: jason@ee.nsysu.edu.tw).

S.-M. Wu is with the Department of Electrical Engineering, National University of Kaohsiung, Kaohsiung 811, Taiwan (e-mail: sungmao@nuk.edu.tw).

J.-Y. Li and C.-C. Chen are with the Information and Communications Laboratories, Industrial Technology and Research Institute, Hsinchu 310, Taiwan (e-mail: jianyu@itri.org.tw).

C.-T. Chiu and C.-P. Hung are with the Research and Development Division, Advanced Semiconductor Engineering, Inc., Kaohsiung 811, Taiwan (e-mail:cp_hung@aseglobal.com).

Color versions of one or more of the figures in this letter are available online at http://ieeexplore.ieee.org.

Digital Object Identifier 10.1109/LMWC.2009.2017593
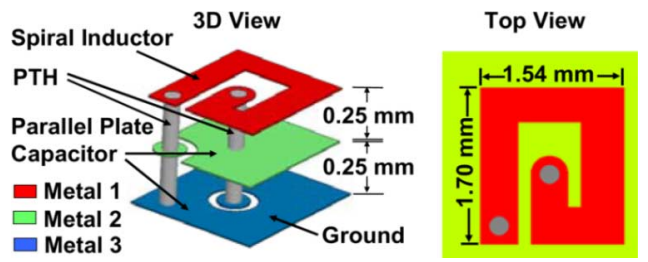

Fig. 1. Stacked $L C$ resonator embedded in a two-layer RT/Duroid 6010 substrate.

with a cross-coupling path always have two transmission zeros near both passband edges, but they are usually limited to a fourth-order design. Lower-order bandpass filters can use the source-load coupling technique [6] to effectively create two transmission zeros on both side of the passband, but they generally take more area to implement. In contrast, the tapped-line feed design [7] can also realize two transmission zeros for bandpass filters without adding too much space. In addition, it can tune the transmission-zero frequencies by varying the tapped position [7]. But a disadvantage of this way is to change the matching condition unless a quarter-wavelength transformer is used as [7].

This letter presents a stacked $L C$ resonator-based bandpass filter embedded in a two-layer substrate that can achieve both miniaturization and transmission-zero creation for $3.5 \mathrm{GHz}$ WiMAX applications. Tapped-line feed and cross-coupling path have been used to create the transmission zeros for secondand fourth-order design. A novel approach is proposed to adjust the transmission-zero frequencies caused by the tapped-line feed. This approach involves etching a rectangular C-shaped slot in the bottom ground plane without adding any space. Controlling the length of the slot can effectively tune the transmission-zero frequencies in the stopband.

\section{Design Methodology}

The presented filter structure comprises coupled resonators embedded in a $0.5 \mathrm{~mm}$ thick two-layer RT/Duroid $6010 \mathrm{sub}-$ strate with dielectric constant of 10.2 and loss tangent of 0.0028 . Fig. 1 shows a single resonator with a dimension of $1.70 \mathrm{~mm}$ $\times 1.54 \mathrm{~mm} \times 0.5 \mathrm{~mm}$. It consists of a spiral inductor on the metal 1 layer and a parallel-plate capacitor using the metal 2 and 3 layers. The inductor and capacitor are stacked as a shunt parallel $L C$ resonator by connecting each other to the metal 3 ground plane with two plated through holes (PTHs). 


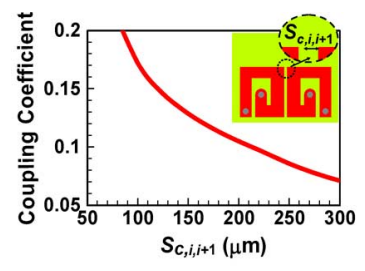

(a)

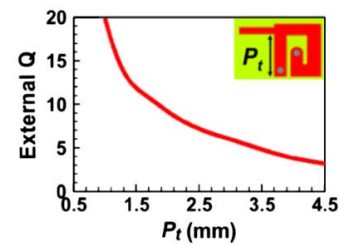

(b)
Fig. 2. (a) Coupling coefficient versus the spacing between two adjacent stacked $L C$ resonators. (b) External quality factor versus the tapped position of the input/output port for a single stacked $L C$ resonator.

The two most important parameters for designing a coupled resonator bandpass filter are the coupling coefficient $k_{i, i+1}$ between two adjacent resonators with indices $i$ and $i+1$ and the external quality factor $Q_{e}$. The coupling coefficient was evaluated as a function of the spacing $\left(S_{c, i, i+1}\right)$ between two adjacent stacked $L C$ resonators, as shown in Fig. 2(a). From knowledge of two dominant resonant frequencies $f_{1}$ and $f_{2}$, the coupling coefficient can be calculated as [8]

$$
k_{i, i+1}= \pm \frac{f_{2}^{2}-f_{1}^{2}}{f_{2}^{2}+f_{1}^{2}} .
$$

The external quality factor of a single stacked $L C$ resonator can be derived in terms of the normalized input admittance $\left(y_{i n}\right)$ and the group delay $\left(\tau_{\Gamma}\right)$ with respect to the reflection coefficient at the resonant angular frequency $\omega_{0}$ [8]. That is

$$
Q_{e}=\frac{\omega_{0} \tau_{\Gamma}\left(\omega_{0}\right)}{4}\left(1-y_{i n}^{2}\left(\omega_{0}\right)\right)
$$

where $y_{i n}$ and $\tau_{\Gamma}$ can be obtained from the reflection coefficient and the derivative of its phase with respect to angular frequency, respectively. As a result, Fig. 2(b) shows the value of $Q_{e}$ as a function of the tapped position $\left(P_{t}\right)$ of the input/output port. Note that the results shown in Fig. 2(a) and (b) are based on full-wave electromagnetic simulation using Ansoft HFSS.

To determine the coupling spacing $\left(S_{c, i, i+1}\right)$ and tapped position $\left(P_{t}\right)$ for an $n$ th-order bandpass filter design, one can use the relations of the $k_{i, i+1}$ and $Q_{e}$ to the filter prototype elements. These relation are given as [8]

$$
\begin{aligned}
k_{i, i+1} & =\frac{\Delta}{\sqrt{g_{i} g_{i+1}}} \\
Q_{e} & =\frac{g_{1}}{\Delta}=\frac{g_{n} g_{n+1}}{\Delta}
\end{aligned}
$$

where $\Delta$ is the fractional bandwidth and $g_{i}$ is the $i$ th prototype element value. For an $n$th order Chebyshev design, the filter passband insertion loss can be predicted by the formula given in [9] and shown as

$$
I L_{\omega_{0}}(d B) \approx \frac{4.343}{\Delta Q_{u}} \sum_{i=1}^{n} g_{i}
$$

where $Q_{u}$ is the unloaded quality factor of a stacked $L C$ resonator at the resonant frequency.

\section{SECOND-ORdER BANDPASS FILTER DESIGN}

A second-order $0.5 \mathrm{~dB}$ equal-ripple Chebyshev bandpass filter is designed at the center frequency of $3.5 \mathrm{GHz}$ with a fractional bandwidth of $17 \%$ and the $Q_{u}$ of a stacked $L C$ resonator

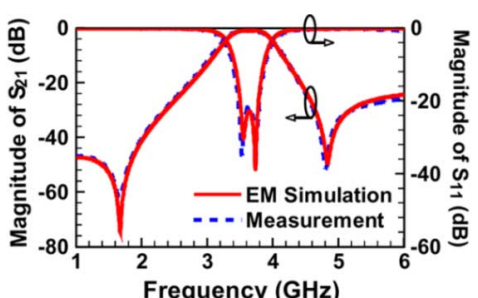

(a)

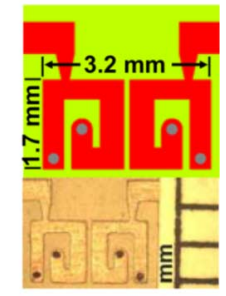

(b)
Fig. 3. Results and structure of the designed second-order bandpass filter. (a) Comparison of the magnitude of $S_{21}$ and $S_{11}$ between electromagnetic simulation and measurement. (b) Top view layout and photograph.

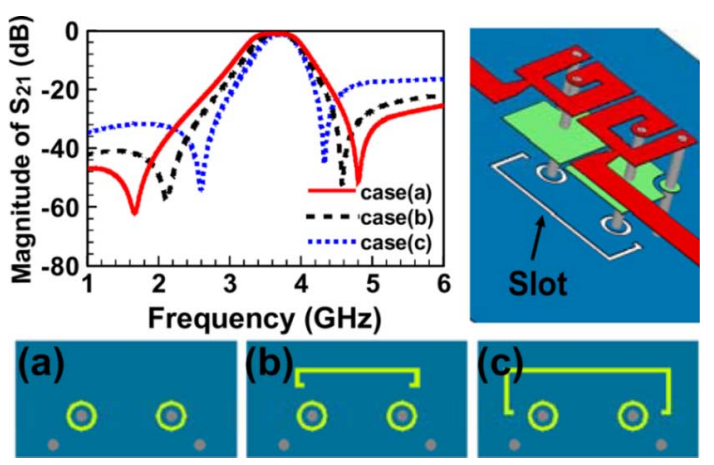

Fig. 4. Comparison of the two transmission-zero frequencies from the measured magnitude of $S_{21}$ for the designed second-order bandpass filter with three different ground structures: Case (a) Without a slot; Case (b) With a $3 \mathrm{~mm}$ long slot; Case (c) With a $5.6 \mathrm{~mm}$ long slot.

is 63. The prototype element values used are $g_{1}=1.403$, $g_{2}=0.707$, and $g_{3}=1.984$. The coupling coefficient $\left(k_{1,2}\right)$ and the quality factor $\left(Q_{e}\right)$ estimated by calculation are 0.17 and 8.25, respectively. It can be found from Fig. 2 that the $k_{1,2}$ and $Q_{e}$ values correspond to a coupling spacing of $S_{c, 1,2}=100 \mu \mathrm{m}$ and a tapped feed position of $P_{t}=2.2 \mathrm{~mm}$. The insertion loss at the center frequency that can be evaluated by (5) is $0.86 \mathrm{~dB}$.

Fig. 3(a) compares the magnitude of $S_{21}$ and $S_{11}$ between electromagnetic simulation and measurement for this secondorder bandpass filter design, showing excellent agreement over the frequency range from 1 to $6 \mathrm{GHz}$. The passband insertion loss is approximately $0.9 \mathrm{~dB}$, which is very close to the predicted value of $0.86 \mathrm{~dB}$, and the passband return loss is larger than $22 \mathrm{~dB}$. The tapped-line feeds shown in Fig. 3(b) can create two transmission zeros at 1.67 and $4.82 \mathrm{GHz}$ on both side of the passband. These two transmission-zero frequencies come from the resonances of the two series resonant paths starting at the tapped position. One path starts from the tapped position, passes through a three-quarter turn spiral segment, and then connects to a parallel-parallel capacitor. The other path chooses to travel through a one-quarter turn spiral segment in another direction and then a PTH to the ground plane. The former path is treated as a series $L C$ resonator, while the latter path is equivalent to a half-wave short circuit. The occupied area of the designed second-order bandpass filter excluding the feedlines is $3.2 \mathrm{~mm}$ $\times 1.7 \mathrm{~mm}$.

This letter also presents a simple approach for tuning the transmission-zero frequencies. The proposed approach etches a rectangular C-shaped slot in the metal 3 ground plane to perturb 


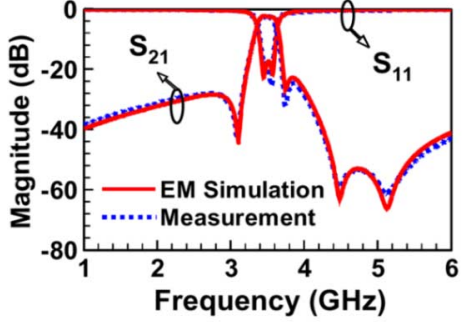

(a)

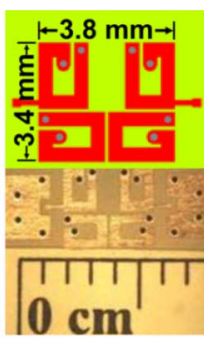

(b)
Fig. 5. Results and structure of the designed fourth-order bandpass filter. (a) Comparison of the magnitude of $S_{21}$ and $S_{11}$ between electromagnetic simulation and measurement. (b) Top view layout and photograph.

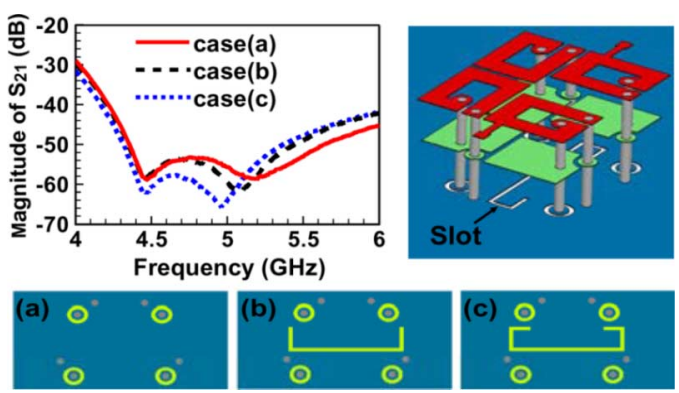

Fig. 6. Comparison of the higher stopband rejection from the measured magnitude of $S_{21}$ for the designed fourth-order bandpass filter with three different ground structures: Case (a) Without a slot; Case (b) With a $4.2 \mathrm{~mm}$ long slot; Case (c) With a $5.2 \mathrm{~mm}$ long slot.

the series resonant paths for causing the transmission zeros. As shown in Fig. 4(b) and (c), different slot lengths on the ground plane result in different transmission-zero frequencies. Case (a) is the original ground plane without a slot. Case (b) and Case (c) shows the ground plane with a $3 \mathrm{~mm}$ and $5.6 \mathrm{~mm}$ long slot, respectively. It can be seen from Fig. 4 that the longer slot pushes the transmission-zero frequencies closer to the passband, causing a higher rolloff rate for passband-to- stopband transition. But it is accompanied by the disadvantage of lowering the minimum rejection level in the stopbands.

\section{FOURTH-ORDER BANDPASS FILTER DESIGN}

Multiple stacked $L C$ resonators can also be cascaded to form a higher order bandpass filter with enhanced selectivity and stopband rejection. In this letter, a fourth-order Chebyshev bandpass filter with $0.01 \mathrm{~dB}$ ripple level is designed with a center frequency of $3.5 \mathrm{GHz}$ and a fractional bandwidth of $10 \%$. The prototype element values used are $g_{1}=0.713, g_{2}=1.20$, $g_{3}=1.321, g_{4}=0.648$, and $g_{5}=1.101$. To reduce the insertion loss of the fourth-order bandpass filter, the $Q_{u}$ of a stacked $L C$ resonator is increased to 76 by widening the metal width of the inductor in the resonator under the same resonant frequency. The estimated coupling coefficients and external quality factor are $k_{1,2}=k_{3,4}=0.108, k_{2,3}=0.079$, and $Q_{e}=7.13$. The insertion loss calculated at the center frequency is $2.22 \mathrm{~dB}$. The required resonator coupling spacing and tapped feed position are found as $S_{c, 1,2}=S_{c, 3,4}=195 \mu \mathrm{m}, S_{c, 2,3}=270 \mu \mathrm{m}$, and $P_{t}=3.1 \mathrm{~mm}$.

As shown in Fig. 5(a), the electromagnetic simulated results are in good agreement with the measured results. In the passband, the measured insertion loss is approximately $2.4 \mathrm{~dB}$, which agrees with the predicted value of $2.22 \mathrm{~dB}$, and the return loss is better than $14.6 \mathrm{~dB}$. Note that the rolloffs on both sides of the passband are very steep because of the two transmission zeros located at 3.09 and $3.74 \mathrm{GHz}$. These two transmission zeros are created by the cross-coupling path. Fig. 5(b) shows the top-view layout and photograph of the designed fourth-order bandpass filter. The tapped-line feeds used at the input and output ports can create two additional transmission zeros at 4.47 and $5.12 \mathrm{GHz}$, which achieves a stopband rejection of more than $45 \mathrm{~dB}$ from 4.3 to $6 \mathrm{GHz}$. The occupied area of the designed fourth-order bandpass filter excluding the feedlines is $3.8 \mathrm{~mm} \times 3.4 \mathrm{~mm}$.

The proposed rectangular C-shaped slots are also implemented on the metal 3 ground plane of the designed fourth-order bandpass filter, as shown in Fig. 6. Case (a), (b) and (c) represents no slot, a $4.2 \mathrm{~mm}$ long slot, and a $5.2 \mathrm{~mm}$ long slot, respectively. It can be seen from Fig. 6 that the longer slot can move the higher two transmission-zero frequencies toward the passband. However, this moving effect is not as obvious as in the second-order design. This is because the passband as well as the lower two transmission zeros near the passband are hardly changed by the presence of the slots, which forms a bigger obstacle to the movement of the higher two transmission zeros.

\section{CONCLUSION}

This letter has presented novel bandpass filter designs by mutually coupling the stacked $L C$ resonators. The advantages Include very small size and ease of implementation using printed circuit technology. The tapped-line feeds used at the input and output ports create two transmission zeros for the proposed bandpass filters. Etching a rectangular C-shaped slot in the bottom ground plane provides an effective way to tune the frequencies of these two transmission zeros. As a result, the presented filter structure can achieve high performance and design flexibility under a stringent size constraint.

\section{REFERENCES}

[1] Q. X. Chu and H. Wang, "A compact open-loop filter with mixed electric and magnetic coupling," IEEE Trans. Microw. Theory Tech., vol. 56, no. 2, pp. 431-439, Feb. 2008.

[2] A. Djaiz and A. Denidni, "A new compact microstrip two-layer bandpass filter using aperture-coupled SIR-hairpin resonators with transmission zeros," IEEE Trans. Microw. Theory Tech., vol. 54, no. 5, pp. 1929-1936, May 2006.

[3] A. Abdel-Rahman, A. R. Ali, S. Amari, and A. S. Omar, "Compact bandpass filters using defected ground structure (DGS) coupled resonators," in IEEE MTT-S Int. Dig., 2005, pp. 12-17.

[4] S.-C. Lin, C.-H. Wang, and C.-H. Chen, "Novel patch-via-spiral resonators for the development of miniaturized bandpass filters with transmission zeros," IEEE Trans. Microw. Theory Tech., vol. 55, no. 1, pp. 137-146, Jan. 2007.

[5] C.-F. Chen, T.-Y. Huang, and R.-B. Wu, "Novel compact net-type resonators and their applications to microstrip bandpass filters," IEEE Trans. Microw. Theory Tech., vol. 54, no. 2, pp. 755-762, Feb. 2006.

[6] D. Ni, Y. Zhu, Y. Xie, and P. Wang, "Synthesis and design of compact microwave filters with direct source-load coupling," J. Electromagn. Waves Appl., vol. 20, no. 13, pp. 1875-1885, 2006.

[7] J.-T. Kuo and E. Shih, "Microstrip stepped impedance resonator bandpass filter with an extended optimal rejection bandwidth,' IEEE Trans. Microw. Theory Tech., vol. 51, no. 5, pp. 1554-1559, May 2003.

[8] J. S. Hong and M. J. Lancaster, Microstrip Filters for RF/Microwave Applications. New York: Wiley, 2001.

[9] R. W. Rhea, HF Filter Design and Computer Simulation. Tucker, GA: Noble Publishing, 1994. 